\title{
Transparent, Flexible, Superomniphobic Surfaces with Ultra-Low Contact Angle Hysteresis**
}

\author{
Kevin Golovin, Duck H. Lee, Joseph M. Mabry, and Anish Tuteja*
}

Engineered surfaces that repel low-surface-tension liquids, such as various oils and alcohols, have a wide array of applications, including stain-proof apparel, fuel transport, chemical shielding, and self cleaning. ${ }^{[1-5]}$ Superomniphobic surfaces display apparent contact angles $\left(\theta^{*}\right)$ that are greater than $150^{\circ}$ and exhibit low contact angle hysteresis (the difference between the advancing and receding contact angles) with essentially all liquids. ${ }^{[5]}$ Previous work has shown how re-entrant curvature is necessary for repelling low-surface-tension liquids. ${ }^{[1,2,6-11]}$ However, it is generally difficult to obtain ultra-low contact angle hysteresis $(\mathrm{CAH}$, $\left.\Delta \theta^{*}<5^{\circ}\right)$ with such low-surface-tension liquids. In our recent work, we discussed the critical role of hierarchical texture in developing superomniphobic surfaces with ultra-low $\Delta \theta^{*}$ values. ${ }^{[1,7]}$ Unfortunately, such hierarchical, superomniphobic surfaces are usually opaque..$^{[1,7,12-17]}$ The development of transparent superomniphobic surfaces is essential for a range of applications, such as coatings for windows, phones, tablets, and computer screens. Herein, we present a facile method for the development of flexible and highly transparent (optical transmission $>90 \%$ ) superomniphobic surfaces that can repel a range of liquids with low or high surface tension.

At the time of writing, only a handful of transparent and omniphobic surfaces had been fabricated..$^{[18-21]}$ None of these surfaces possesses hierarchical texture, which yields ultra-low $\Delta \theta^{*}$ values. In most of the previous studies, transparency was only achieved at the cost of lower $\theta^{*}$ and/or higher $\Delta \theta^{*}$ values for low-surface-tension liquids. Now, using previously developed design parameters, ${ }^{[10]}$ we were able to tune the texture of our surfaces to develop one of the first transparent superomniphobic surfaces with ultra-low contact angle hysteresis.

When a liquid comes in contact with a solid surface, the system can either adopt the fully wetted Wenzel state ${ }^{[22]}$ or yield a composite liquid-air interface, which is known as the

[*] K. Golovin, Dr. D. H. Lee, Prof. A. Tuteja

Department of Materials Science and Engineering

University of Michigan

Ann Arbor, MI 48109 (USA)

E-mail: atuteja@umich.edu

Dr. J. M. Mabry

Space and Missile Propulsion Division

Air Force Research Laboratory

Edwards Air Force Base, CA 93524 (USA)

[**] We thank Dr. Charles Y.-C. Lee and the Air Force Office of Scientific Research (AFOSR) for financial support (FA9550-11-1-0017 and LRIR-12RZ03COR). We also thank the Office of Naval Research (ONR) for financial support (N00014-12-1-0874).

(6) Supporting information for this article is available on the WWW under http://dx.doi.org/10.1002/anie.201307222.
Cassie-Baxter state. ${ }^{[23]}$ A robust Cassie-Baxter state is required for superomniphobicity, because surfaces in the Wenzel state that yield contact angles $\theta^{*}>150^{\circ}$ have not been developed for low-surface-tension liquids. Typically, the wetted Wenzel state is thermodynamically favorable for low-surface-tension liquids. Accordingly, the rational design of superomniphobic surfaces that repel low-surface-tension liquids should focus on keeping the metastable Cassie-Baxter state as robust as possible.

Surfaces that are textured on multiple length scales are beneficial for ultra-low $\mathrm{CAH}$, because CAH strongly depends on the solid-liquid contact area. ${ }^{[1,5,7]}$ Two dimensionless parameters can be useful in the design of superomniphobic surfaces. ${ }^{[10]} D^{*}$ is a measure of the solid-liquid interfacial area, and for our surfaces, $D^{*}=[(R+D) / R]^{2}$ where $2 R$ is the diameter of the texture, and $2 D$ is the inter-feature spacing (Figure 2B). As $D^{*}$ increases, the liquid comes in contact with less of the solid surface, which leads to an increase in the $\theta^{*}$ value. The Cassie-Baxter relationship can be rewritten in terms of $D^{*}$ [Eq. (1)]. ${ }^{[7]}$

$\cos \left(\theta^{*}\right)=-1+\frac{1}{D^{*}}\left[\frac{\pi}{2 \sqrt{3}}(1+\cos \theta)\right]^{2}$

$\theta$ is the contact angle given by Young's relation. ${ }^{[24]}$ Examining this equation gives an apparent contact angle approaching $180^{\circ}$ when $D^{* \gg 1}$, because with increasing $D^{*}$, the contact area of the liquid with air increases. The other critical design parameter, the robustness factor $A^{*}$, is a measure of the stability of the Cassie-Baxter state for a given surface. ${ }^{[10]} A^{*}$ is a confluence of the sagging angle and sagging depth of a liquid droplet and can be written as $P_{\mathrm{b}} / P_{\text {ref }}$, where $P_{\mathrm{b}}$ is the breakthrough pressure that forces the surface to transition to the Wenzel state, and $P_{\text {ref }}$ is a reference pressure given by $P_{\text {ref }}=2 \gamma_{\mathrm{lv}} / l_{\text {cap }}$, with a capillary length $l_{\text {cap }}=\left[\gamma_{\mathrm{lv}} / \rho g\right]^{1 / 2}$. Here, $\gamma_{\mathrm{lv}}$ is the liquid surface tension, $\rho$ is the liquid density, and $g$ is the acceleration that is due to gravity. ${ }^{[10]}$ Considering a twodimensional geometry of discrete spherical particles, the robustness factor $A^{*}$ can be written as Eq. (2) $)^{[7]}$ (for an expression for $A^{*}$ on pillar-like structures, see the Supporting Information, Section 2).

$A^{*}=\frac{P_{\mathrm{b}}}{P_{\text {ref }}}=\frac{2 \pi \ell_{\text {cap }}}{R_{\text {particle }}\left(2 \sqrt{3} D^{*}-\pi\right)} \frac{(1-\cos \theta)}{\left(\sqrt{D^{*}}-1+2 \sin \theta\right)}$

When $A^{*}<1$, the Cassie-Baxter state is unstable, and the composite interface cannot be supported. Using this understanding, we herein designed surfaces for which both $D^{*}$ and $A^{*} \gg 1$. 
Transparent, flexible, superomniphobic surfaces were created using a facile spray method on master-molded polydimethyl siloxane (PDMS) pillars. Briefly, silicon master molds with a uniform square array of holes $(2 R=$ $15 \pm 0.2 \mu \mathrm{m})$ were fabricated using standard photolithography. We prepared molds with several $D^{*}$ values ranging from 10-100 (corresponding $A^{*}$ values from 27 to 0.8 with water). PDMS was chosen as the pillar material because it is highly transparent, flexible, and possesses a low surface energy. ${ }^{[18]}$ Pillar heights of 20 and $40 \mu \mathrm{m}$ were chosen for this work. Pillars were subsequently sprayed with solutions of $1 H, 1 H, 2 H, 2 H$-heptadecafluorodecyl polyhedral oligomeric silsesquioxane (F-POSS), ${ }^{[9,25]}$ along with more PDMS as a binder. The high degree of fluorination makes F-POSS one of the lowest-surface-energy molecules available $\left(\gamma_{\mathrm{sv}} \approx 10 \mathrm{mN} \mathrm{m}^{-1}\right)$. Spraying low-surface-energy polymers can lead to a controllable re-entrant texture with oleophobic properties, as explained previously. ${ }^{[12]}$ The synthesis method is shown in Figure $1 \mathrm{~A}$. The photolithography necessary to create the master mold only needs to be performed once (for experimental details, see the Supporting Information).

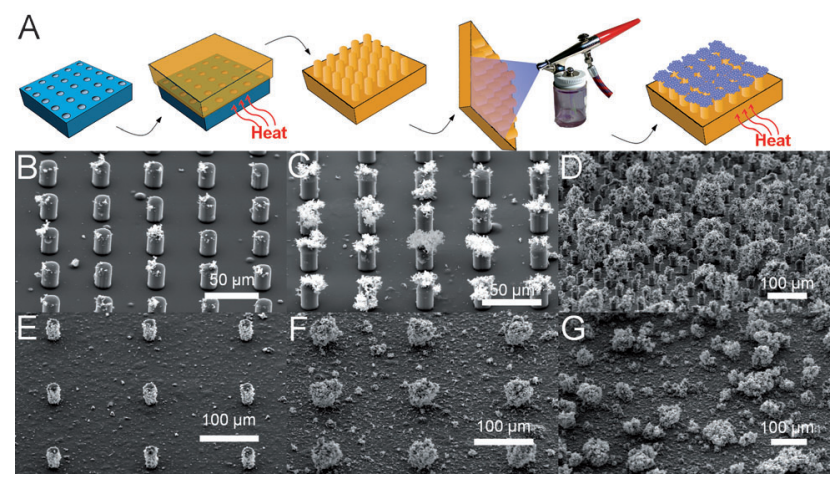

Figure 1. A) Fabrication method. First, the desired pattern is etched into a silicon master mold using photolithography. PDMS is poured into the master mold and cured in an oven. PDMS/F-POSS is sprayed onto the pillars, which are subsequently cured again. B-D) PDMS pillars $\left(D^{*}=10\right)$ spray-coated with PDMS/F-POSS for 30,120 , and 240 seconds. E-G) PDMS Pillars with $D *=100$ spray-coated for 30 , 120 , and 180 seconds.

SEM micrographs of two representative surfaces with $D^{*}$ values of 10 and 100 that were sprayed with the PDMS/FPOSS system for various spray times are shown in Figure 1 BG. Without any sprayed solution (Supporting Information, Figure $\mathrm{S} 1$ ), only the surface with $D^{*}=10$ is robustly superhydrophobic, and neither of the surfaces are superomniphobic (as they lack re-entrant curvature). This matches well with the calculated $A^{*}{ }_{\text {water }}$ values of 26.8 and 0.84 and the calculated $A^{*}{ }_{\text {ethanol }}$ values of 0.37 and 0.009 for $D^{*}$ values of 10 and 100 , respectively. In two recent publications, discrete pillars were fabricated with re-entrant curvature on the macroscale. ${ }^{[18,19]}$ However, without hierarchical texture, these surfaces have relatively high $\mathrm{CAH}\left(\Delta \theta^{*}>5^{\circ}\right)$ for low-surfacetension liquids. The spray-coating-based method described herein introduces both re-entrant curvature (overhang of the
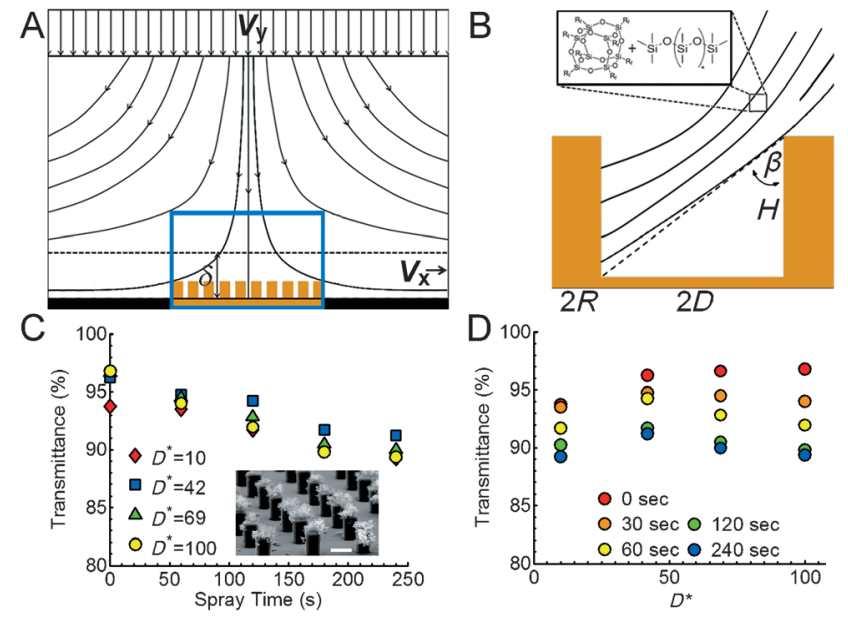

Figure 2. A) Stagnation point flow created by our spray setup ( $\delta=$ boundary layer thickness). The corresponding streamlines are also shown. The blue box denotes the applicable region for our setup, where substrates are directly beneath the axis of the spray gun. B) A model elucidating how particle deposition can be localized only onto the top and the side walls of the PDMS pillars. The $\beta$ value shown is for an ideal case where particles reach the bottom of the pillars, but not the region in between the pillars $\left(\beta=\beta_{\mathrm{cr}}\right)$. The pillars alone lack reentrant curvature and cannot be oleophobic. C-D) Transparency of the surfaces coated with the PDMS/F-POSS spray for different spray times and $D *$ values. For all spray times greater than 0 seconds, a maximum in transparency persists at $D *=42$.

sprayed aggregates atop the pillars) and a hierarchical texture, which leads to ultra-low CAH.

The spray method was chosen because it creates a unique flow field that can localize particle deposition, which helps us to fabricate hierarchically textured surfaces. When the spray gun is pointed directly down (or along) the pillar axes (Figure 2A), an axisymmetric stagnation-point flow field is created. This type of flow field is well-characterized and has a boundary layer thickness $\delta$, which may be described by Eq. (3). ${ }^{[26]}$

$\delta=2.4 \sqrt{\frac{v}{a}}$

Here, $v$ is the kinematic viscosity, and $a$ is a constant arising from the dimensionless velocity profile $\left(V_{y}=-a y\right) \cdot{ }^{[26]}$ Our flow field yielded a boundary layer thickness of $\delta=94 \pm$ $5 \mu \mathrm{m}$ (see the Supporting Information, Section 4). Physically, a boundary layer thickness of this scale implies that any height up to approximately $100 \mu \mathrm{m}$ from the surface will have a significant velocity component that is perpendicular to the pillar axis $\left(V_{x}\right)$, even though the spray setup is pointed along the pillar axis. Beyond $\delta, 99 \%$ of the velocity profile matches the mainstream flow (along the pillar axis).

A two-dimensional model elucidates how the horizontal velocity component $\left(V_{x}\right)$ can lead to controllable and localized particle deposition (Figure 2B). Particles within the streamlines of the flow approach the pillar tops at an angle $\beta$. Considering a pillar height $H$ and an inter-feature spacing $2 D$, the critical angle required for a streamline to intersect the bottom of a pillar is given by Eq. (4). 
$\beta_{\text {cr }}=\tan ^{-1}\left(\frac{2 D}{H}\right)$

Typically, $\beta$ is fixed for a set of given spray conditions; in our experiments, however, the pillar spacing was varied over a wide range. For a given pillar height and spacing, values of $\beta<\beta_{\text {cr }}$ will cause streamlines to intersect the substrate in between the pillars rather than to hit the pillar sidewalls.

The effects of spray time and $D^{*}$ on the transparency of the fabricated surfaces are illustrated in Figure $2 \mathrm{C}$ and D. As the fraction of pillars per unit area decreases (increasing $D^{*}$ ), the transmittance increases, as expected. The deviations in transmission from the values obtained for a smooth PDMS film originate from the slight scattering that is caused by the pillar edges; this effect amplifies with increasing pillar density. For a sample with $D^{*} \approx 1$ (Figure S2 A), the transparency is lower than $60 \%$. As the spray time increases, the transparency decreases, because a higher fraction of the surface is covered with the opaque PDMS/F-POSS particles. For our spray conditions, the highest transparency occured at $D^{*}=42$ ( $R=15,2 D=82$; Figure 2D). With our model, a maximum in transparency can be expected: By increasing $D^{*}$ we can reduce the scattering caused by the pillar edges; however, this also increases the probability of particles aggregating in between the pillars.

Assuming that $\beta=\beta_{\text {cr }}$ for pillars with $D^{*}=42$, for a pillar height of $20 \mu \mathrm{m}$, a $\beta$ value of $76^{\circ}$ can be estimated. Physically, this means that if the pillar spacing is varied so that $D^{*}<42$, all incoming particles will aggregate on a pillar wall, rather than land in between the pillars. For $D^{*}>42$ (or $\beta<\beta_{\text {cr }}$ ), particles will aggregate on the pillar walls as well as in between the pillars. According to our model, for the geometry $\left(D^{*}\right)$ with optimized transparency, all particles with a velocity component that is perpendicular to the pillar axis will stick to a pillar wall rather than land in the regions between the pillars. Other geometries can then be divided into two regimes: $\beta<\beta_{\text {cr }}$ and $\beta>\beta_{\text {cr }}$. For our surface with $D^{*}=10$, a $\beta_{\text {cr }}$ value of $58^{\circ}$ can be calculated. Although the spacing between the pillars $(2 D)$ has changed, the spraying conditions are equivalent to the $D^{*}=42$ case, and thus $\beta$ is still $76^{\circ}$. Back-solving for the height of the pillars at minimum incidence yields a value of $8 \mu \mathrm{m}$ (Figure S3), which implies that as our pillars are $20 \mu \mathrm{m}$ in height, only the top $12 \mu \mathrm{m}$ will collect particles, which leads to controllable particle deposition. This model is supported by the SEM micrographs in Figure $1 \mathrm{~B}-\mathrm{D}$ and Figure $3 \mathrm{C}$ (inset); the majority of the PDMS/F-POSS aggregates sit atop the PDMS pillars. A similar analysis can be performed for a geometry with $D^{*}=$ 100 . In this case, the value of $\beta_{\text {cr }}$ is $82^{\circ}$; therefore, particles will not only stick to the pillar walls, but also to the space in between the pillars (Figure 1E-G). The re-entrant curvature produced by particles sitting atop the pillars and on the pillar side walls is necessary to repel liquids where the equilibrium contact angle is $<90^{\circ}$. For example, spraying the same solution onto a flat PDMS substrate (not shown) yields oleophilic surfaces, with $\theta^{*}=22^{\circ}$ and $28^{\circ}$ for ethanol and hexadecane, respectively.

Once high transparency had been achieved through localized particle deposition, we evaluated the liquid repel-
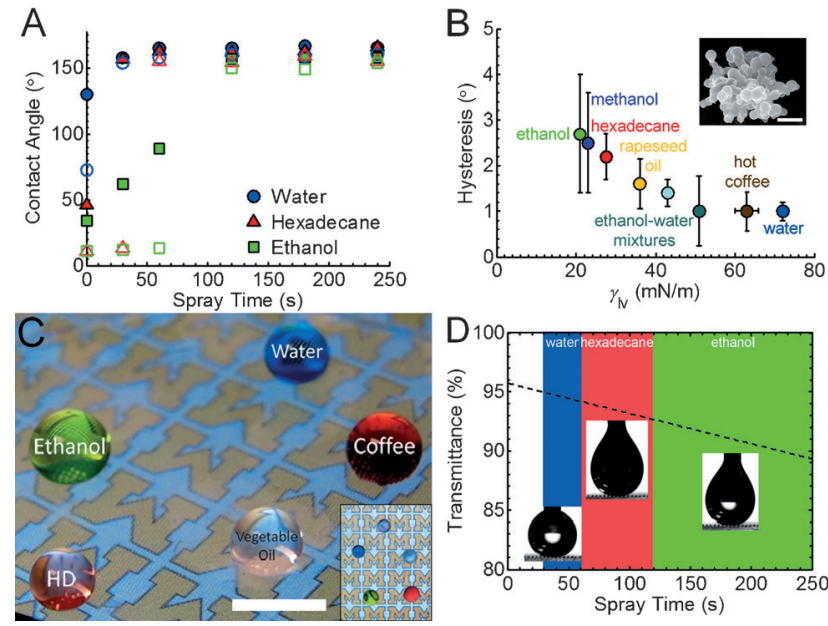

Figure 3. $\mathrm{A}, \mathrm{B}$ ) Contact angle measurements with water, hexadecane, and ethanol for pillars with $D^{*}=100$. The filled and open symbols represent advancing and receding contact angles, respectively. The surface in (B) was sprayed for 120 seconds. The inset shows the reentrant PDMS/F-POSS structure; scale bar: $5 \mu \mathrm{m}$. C) Droplets of varying surface tension beading up on an Apple iPhone 3GS screen coated with $20 \mu \mathrm{m}$ pillars with $D^{*}=42$, and spray coated with PDMS/ F-POSS for 120 seconds. The inset shows the top view of the same droplets to highlight the transparency of the coating. Scale bar: $500 \mu \mathrm{m}$. D) A design diagram for pillars with $D *=100$ combining transparency and repellency characteristics. The insets are optical images of droplets of water, hexadecane (HD), and ethanol sitting atop the spray-coated pillars. The composite interface is clearly visible underneath the liquid droplets.

lency of our surfaces. For our experiments, we chose hexadecane (nonpolar liquid) and ethanol (polar) as representative low-surface-tension liquids $\quad\left(\gamma_{\mathrm{lv}}=27.5\right.$ and $21.8 \mathrm{mN} \mathrm{m}^{-1}$, respectively). ${ }^{[27,28]}$ The advancing and receding contact angles were measured versus spray time for a pattern with $D^{*}=100$ (Figure $3 \mathrm{~A}$ ).

As stated earlier, without spraying the surface, the $A^{*}$ water value is 0.84 . After spraying with the PDMS/F-POSS solution for 30 seconds, the composite interface can be supported for water, but not for hexadecane $\left(A^{*}{ }_{\text {water }}=1.94 ; A^{*}{ }_{\text {hexadecane }}=\right.$ 0.94). After a spray time of $60 \mathrm{sec}$, hexadecane can exist in the Cassie-Baxter state $\left(A^{*}{ }_{\text {hexadecane }}=1.85 ; A^{*}{ }_{\text {ethanol }}=0.92\right)$, and after 120 seconds, ethanol is also repelled by the surface $\left(A^{*}{ }_{\text {ethanol }}=1.04\right)$. For patterns with $D^{*}=10$, water is stable in the Cassie-Baxter state without any spray because of an $A^{*}{ }_{\text {water }}$ value of 26.8 , whereas ethanol is repelled after a spray time of approximately 45 seconds (for all $A^{*}$ values, see Table S1). For low $D^{*}$ values, low-surface-tension liquids exhibit larger $\mathrm{CAH}$ for all tested spray times $\left(\Delta \theta^{*}>10^{\circ}\right)$, owing to the increased solid fraction $\left(\phi_{\mathrm{s}} \approx 8 \%\right)$. CAH for pillars with $D^{*}=100$ that were sprayed for 120 seconds is shown as a function of liquid surface tension in Figure $3 \mathrm{~B}$. Even for liquids with very low surface tension, such as ethanol, the hysteresis $\Delta \theta^{*}$ is smaller than $3^{\circ}$.

An iPhone 3GS screen that was coated with one of our surfaces $\left(D^{*}=42\right.$, spray time $\left.=120 \mathrm{~s}\right)$ repelled different liquids with a range of surface tensions (Figure $3 \mathrm{C}$ ). The inset is a top view of the screen. It is evident that our surfaces are not only mathematically transparent (i.e., there is a small 
percentage of sparsely located opaque pillars), but also visually transparent, because the pillars are invisible to the naked eye. With our control over $D^{*}$, re-entrant curvature, and hierarchical texture, a design diagram was constructed for coatings with $D^{*}=100$ (Figure $3 \mathrm{D}$ ). This diagram combines aspects of liquid repellency, transparency, and spray time into a single plot. It is then trivial to select the desired surface tension liquid that is to be to repelled and to predict the final transparency of the substrate. Similar design diagrams can be constructed for other $D^{*}$ values.

We also evaluated the effects of varying the pillar height on the transparency of the fabricated surfaces. With increasing pillar height, the PDMS/F-POSS particles were found to agglomerate more quickly around the pillars because of the increase in surface area (and decreasing $\beta_{\text {cr }}$ ). To better understand this phenomenon, spray coating was combined with time-lapsed optical microscopy for different pillar heights and $D^{*}$ values over a spray time period of 240 seconds with intervals of 15 seconds. In Figure $4 \mathrm{~A}$, frames (from the

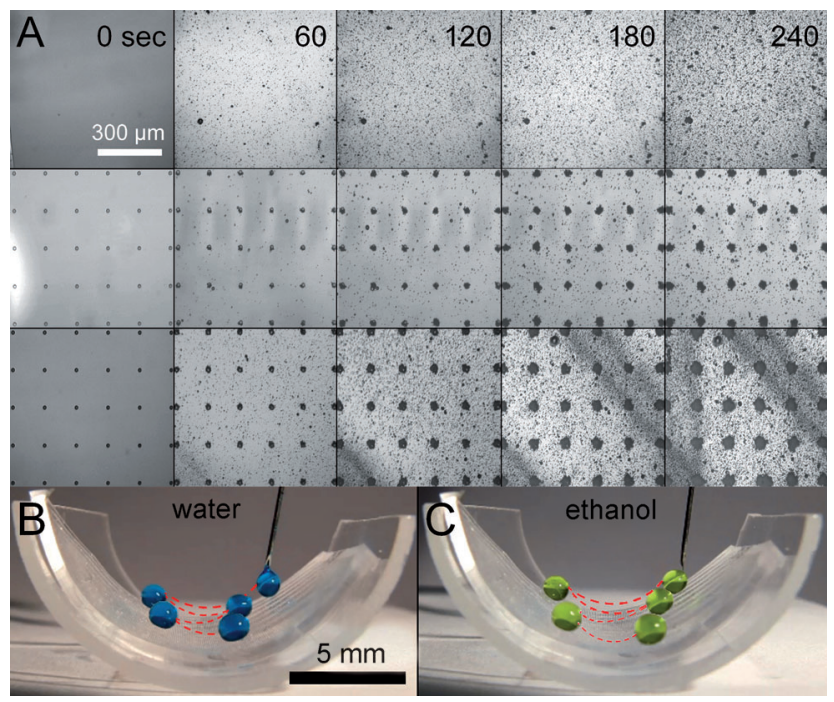

Figure 4. A) Time-lapsed movie frames corresponding to flat PDMS (top), $20 \mu \mathrm{m}$ high pillars with $D^{*}=100$ (middle), and $40 \mu \mathrm{m}$ high pillars with $D^{*}=100$ (bottom). The frames support the proposed model, because fewer particles accumulate between the $20 \mu \mathrm{m}$ high pillars than between the $40 \mu \mathrm{m}$ high pillars. B, C) Movie frames taken with $10 \mathrm{~ms}$ intervals are superimposed for droplets of water and ethanol, respectively. The droplets roll back and forth several times before reaching the edge of the substrate, which highlights the ultralow $\mathrm{CAH}$.

movies M1, M2, and M3; see the Supporting Information) are shown that correspond to flat PDMS, pillars with $D^{*}=100$ and $H=20 \mu \mathrm{m}$, and pillars with $D^{*}=100$ and $H=40 \mu \mathrm{m}$, respectively. It should be emphasized that all surfaces were sprayed together within $5 \mathrm{~mm}$ of one another, so that the effects seen here are purely due to geometry. It is evident that a greater pillar height leads to faster lateral growth of the PDMS/F-POSS aggregates, which in turn lowers the overall transparency. However, because of the small pillar size $(2 R=$ $15 \mu \mathrm{m})$ and sparse pillar density $(2 D=140 \mu \mathrm{m})$, the optimally sprayed surfaces are visually still close to a transparency of $100 \%$.

The low CAH and the flexibility of our fabricated surfaces are highlighted in Movie M4. The fabricated surfaces with $D^{*}=100$ (sprayed for 120 seconds) were curved into arcs, and various droplets of liquids with high or low surface tension were deposited on them. The droplets roll back and forth along the length of the surface until the edge is reached. The trajectories of both water and ethanol molecules are shown in the superimpositions of $10 \mathrm{~ms}$-spaced frames (Figure 4B,C). To the best of our knowledge, this is one of the first flexible and transparent surfaces that display ultra-low contact angle hysteresis with low-surface-tension liquids, such as ethanol. Furthermore, the simple mold and spray fabrication method is well-suited for scale-ups to functionalize significantly larger areas.

Received: August 16, 2013

Revised: October 3, 2013

Published online: November 13, 2013

Keywords: materials science $\cdot$ spray methods .

superomniphobicity · surface chemistry · transparency

[1] S. Pan, A. K. Kota, J. M. Mabry, A. Tuteja, J. Am. Chem. Soc. 2013, 135, 578-581.

[2] W. Choi, A. Tuteja, S. Chhatre, J. M. Mabry, R. E. Cohen, G. H. McKinley, Adv. Mater. 2009, 21, 2190-2195.

[3] B. Bhushan, Y. C. Jung, Prog. Mater. Sci. 2011, 56, 1-108.

[4] H. Jin, M. Kettunen, A. Laiho, H. Pynnonen, J. Paltakari, A. Marmur, O. Ikkala, R. H. Ras, Langmuir 2011, 27, 1930-1934.

[5] A. K. Kota, W. Choi, A. Tuteja, MRS Bull. 2013, 38, 383-390.

[6] A. Ahuja, J. A. Taylor, V. Lifton, A. A. Sidorenko, T. R. Salamon, E. J. Lobaton, P. Kolodner, T. N. Krupenkin, Langmuir 2008, 24, 9-14.

[7] A. K. Kota, Y. Li, J. M. Mabry, A. Tuteja, Adv. Mater. 2012, 24, $5838-5843$

[8] A. Marmur, Langmuir 2008, 24, 7573-7579.

[9] A. Tuteja, W. Choi, M. Ma, J. M. Mabry, S. A. Mazzella, G. C. Rutledge, G. H. McKinley, R. E. Cohen, Science 2007, 318, $1618-1622$.

[10] A. Tuteja, W. Choi, J. M. Mabry, G. H. McKinley, R. E. Cohen, Proc. Natl. Acad. Sci. USA 2008, 105, 18200-18205.

[11] L. Cao, T. P. Price, M. Weiss, D. Gao, Langmuir 2008, 24, $1640-$ 1643.

[12] S. Srinivasan, W. Choi, K.-C. Park, S. S. Chhatre, R. E. Cohen, G. H. McKinley, Soft Matter 2013, 9, 5691-5702.

[13] S. P. Kobaku, A. K. Kota, D. H. Lee, J. M. Mabry, A. Tuteja, Angew. Chem. 2012, 124, 10256-10260; Angew. Chem. Int. Ed. 2012, 51, 10109-10113.

[14] J. Yang, Z. Zhang, X. Xu, X. Men, X. Zhu, X. Zhou, New J. Chem. 2011, 35, 2422.

[15] T. P. Nhung Nguyen, P. Brunet, Y. Coffinier, R. Boukherroub, Langmuir 2010, 26, 18369-18373.

[16] C. H. Xue, P. Zhang, J. Z. Ma, P. T. Ji, Y. R. Li, S. T. Jia, Chem. Commun. 2013, 49, 3588-3590.

[17] S. S. Chhatre, A. Tuteja, W. Choi, A. Revaux, D. Smith, J. M. Mabry, G. H. McKinley, R.E. Cohen, Langmuir 2009, 25, 13625-13632.

[18] S. M. Kang, S. M. Kim, H. N. Kim, M. K. Kwak, D. H. Tahk, K. Y. Suh, Soft Matter 2012, 8, 8563-8568.

[19] S. E. Lee, H. J. Kim, S. H. Lee, D. G. Choi, Langmuir 2013, 29, $8070-8075$. 
[20] J. Zhang, S. Seeger, Angew. Chem. 2011, 123, 6782-6786; Angew. Chem. Int. Ed. 2011, 50, 6652-6656.

[21] X. Deng, L. Mammen, H. J. Butt, D. Vollmer, Science 2012, 335, $67-70$.

[22] R. N. Wenzel, Ind. \& Engg. Chem. 1936, 28, 988; Engg. Chem. 1936, 28, 988.

[23] A. B. D. Cassie, S. Baxter, Trans. Faraday Soc. 1944, 40, $546-$ 551.

[24] T. Young, Philos. Trans. R. Soc. London 1805, 95, 65-87.
[25] J. M. Mabry, A. Vij, S. T. Iacono, B. D. Viers, Angew. Chem. 2008, 120, 4205-4208; Angew. Chem. Int. Ed. 2008, 47, 41374140.

[26] F. M. White, Viscous fluid flow, McGraw-Hill, New York, 1991.

[27] E. A. Gonzalo Vhquez, J. M. Navaza, J. Chem. Eng. Data 1995, 40, 611-614.

[28] G. Kwon, A. K. Kota, Y. Li, A. Sohani, J. M. Mabry, A. Tuteja, Adv. Mater. 2012, 24, 3666-3671. 